\title{
PRODUCCIÓN Y CONTENIDO NUTRIMENTAL EN AVENA FORRAJERA FERTILIZADA CON FUENTES QUÍMICAS Y ORGÁNICAS
}

\author{
PRODUCTION AND NUTRITIONAL CONTENT IN FORAGE OATS \\ FERTILIZED WITH CHEMICAL AND ORGANIC SOURCES
}

\section{Mario Montaño-Carrasco ${ }^{1}$, Adriana Hernández-Rodríguez ${ }^{1}$, Andrés Martínez-Rosales ${ }^{2}$, Dámaris Ojeda-Barrios ${ }^{1}$, Abelardo Núñez-Barrios ${ }^{1}$ y Víctor Guerrero-Prieto ${ }^{1}$}

Ciencias Agrotecnológicas, Universidad Autónoma de Chihuahua. Ciudad Universitaria s/n Campus 1. 31310, Chihuahua, Chihuahua, México. Tel. (614) 4391844 Ext. 3127. ${ }^{2}$ Campus Puerto Ángel, Universidad Del Mar. 70902, Oaxaca, México.

*Autor para correspondencia (aernande@uach.mx)

\section{RESUMEN}

La fertilización es un componente fundamental en la producción de cultivos y en la calidad de las cosechas; además, deja secuelas en el sustrato en que se aplica. El objetivo de este estudio fue comparar el efecto de la fertilización orgánica, inorgánica y su combinación sobre la producción y contenido mineral del forraje de avena (Avena sativa L.) y en las propiedades fisicoquímicas del suelo. El estudio se realizó en Namiquipa, Chihuahua, México en el año 2015. Se utilizó un diseño experimental completamente al azar para evaluar cinco tratamientos: 1) T1-C: fertilización orgánica con el uso de compost, 2) T2-L: fertilización orgánica con lixiviados de vermicompost, 3) T3-Q: fertilización química mediante dos aplicaciones, de $18 \mathrm{~N}-46 \mathrm{P}-00 \mathrm{~K}$ y $46 \mathrm{~N}-00 \mathrm{P}-00 \mathrm{~K}, 4)$ T4-QC: fertilización química más compost, y 5) T5-QL: fertilización química más lixiviados de vermicompost. Las variables evaluadas en suelo fueron: materia orgánica, densidad aparente, conductividad eléctrica, macro y micronutrientes; en planta: densidad de planta, producción de materia seca, macro y micronutrientes. Se realizó análisis de varianza y comparación de medias con la prueba de Tukey $(P \leq 0.05)$ mediante el paquete estadístico SAS. Los resultados mostraron que el tratamiento de compost (T1-C) arrojó los mejores valores de las variables evaluadas en suelo y un mayor incremento en el contenido de materia orgánica con $18.1 \mathrm{~g} \mathrm{~kg}^{-1}$, seguido por el tratamiento de lixiviado de vermicompost (T2-L) con $17.3 \mathrm{~g} \mathrm{~kg}^{-1}$. Los valores más altos de peso seco y contenido de nitrógeno se observaron en el forraje de avena obtenida con los tratamientos T3-Q, T4-QC y T5-QL. Se observaron diferencias en el contenido nutrimental del forraje con los diferentes tipos de fertilización, con excepción en $\mathrm{Na}$, $\mathrm{Cu}$ y $\mathrm{Zn}$, donde los tratamientos T3-Q y T5-QL fueron los que sobresalieron en el aporte de nutrientes. Con base en los resultados, se recomienda adicionar abonos orgánicos al suelo, ya que mejoran diversos indicadores de fertilidad durante el mismo ciclo, además de reflejar una buena combinación con la fertilización química en la producción de avena forrajera.

Palabras clave: Compost, lixiviados, vermicompost, fertilización combinada.

\section{SUMMARY}

Fertilization is a key component in crop production and harvest quality; in addition, it leaves sequels in the substrate where it is applied. The objective of this study was to compare the effect of organic and inorganic fertilization and their combination, on the production and mineral content of oat (Avena sativa L.) fodder, and on the physico-chemical properties of the soil. The study was performed in Namiquipa, Chihuahua, Mexico in 2015. A completely randomized experimental design was used to evaluate five treatments: 1)
T1-C: organic fertilization using compost, 2) T2-L: organic fertilization with vermicompost leachate, 3) T3-Q: chemical fertilization in two applications, $18 \mathrm{~N}-46 \mathrm{P}-00 \mathrm{~K}$ and $46 \mathrm{~N}-00 \mathrm{P}-00 \mathrm{~K}, 4) \mathrm{T} 4-\mathrm{QC}$ : chemical fertilization plus compost, and 5) T5-QL: chemical fertilization plus vermicompost leachate. The variables evaluated in soil were: organic matter, apparent density, electrical conductivity, macro and micronutrients; in plant: plant density, dry matter production, macro and micronutrients. Analysis of variance and means comparison using the Tukey test $(P \leq 0.05)$ were performed through the SAS statistical package. Results showed that compost treatment (T1-C) produced the best values of the evaluated variables in soil and a greater increase in organic matter with $18.1 \mathrm{~g} \mathrm{~kg}^{-1}$, followed by the treatment of vermicompost leachate (T2-L) with $17.3 \mathrm{~g} \mathrm{~kg}^{-1}$. The highest values of dry weight and nitrogen content were observed in the oat fodder obtained with T3-Q, T4-QC and T5$\mathrm{QL}$ treatments. Differences were observed in nutritional content of the forage with the different types of fertilization, except for $\mathrm{Na}, \mathrm{Cu}$ and $\mathrm{Zn}$, where T3-O and T5-QL treatments were the ones that excelled in nutrient supply. Based on the results, it is recommended to add organic fertilizers to the soil, as they improve various indicators of fertility during the same cycle, in addition to reflecting a good combination with chemical fertilization in the production of fodder oats.

Index words: Compost, leachate, vermicompost, combined fertilization.

\section{INTRODUCCIÓN}

La avena (Avena sativa L.) es una planta importante como productora de grano, que también se utiliza como forraje para la alimentación de animales en pastoreo, heno o ensilado (Ramírez-Ordóñes et al., 2013). El estado de Chihuahua es el principal productor de avena en México, con una participación del 33 \% del producto. y es cultivada extensamente bajo condiciones de temporal y riego (SIAP, 2015). Esta gramínea es la base del sistema de producción regional de leche, y representa el 71 \% del costo total de alimentación del ganado; también se usa en los sistemas extensivos de bovinos productores de carne (Jurado et al., 2015, Com. Pers' ${ }^{1}$ )

'Jurado G. P., C. R. Lara M. y J. S. Sierra T. (2015) Guía técnica para la producción de avena forrajera en Chihuahua. $15 \mathrm{p}$. http://siproduce. sifupro.org.mx/seguimiento/archivero/8/2013/trimestrales/ anexo_2413-5-2014-02-2.pdf 
La concentración nutrimental en el forraje es esencial para el crecimiento y reproducción de animales. Los macronutrientes, elementos requeridos en grandes cantidades, son componentes importantes de huesos y tejidos, sirven como constituyentes de fluidos corporales y juegan roles vitales en las funciones metabólicas. Los micronutrientes están presentes en los tejidos del cuerpo en muy bajas concentraciones y frecuentemente sirven como componentes de metaloenzimas y cofactores enzimáticos. Por su importancia en la nutrición del ganado, el análisis de laboratorio de los macro y micronutrientes contenidos en los alimentos es necesario para precisar la formulación de dietas a fin de satisfacer los requerimientos a bajo costo (NRC, 2001).

Para la producción de avena se ha hecho uso, durante muchos años, de los paquetes tecnológicos de la agricultura intensiva, hasta el punto en el que la aplicación de los fertilizantes inorgánicos se ha convertido en un problema ambiental en muchos lugares del mundo (Butler et al., 2007). Estas prácticas, aunadas a las grandes cantidades de residuos orgánicos (RO) generados por las actividades agrícolas, provocan severos daños al ecosistema (Flotats y Solé, 2008); por esta razón, se ha considerado que los procesos de descomposición controlada deben ser actividades obligatorias aplicadas a los RO para contribuir en la reducción de los riesgos (Lübke y Lübke, 2008) y obtener productos aprovechables en la agricultura (Fornes et al., 2012).

Actualmente existe un importante interés por encontrar alternativas de fertilización a partir del compostaje (CP) y para vermicpompostaje (VC) (Márquez-Hernández et al., 2013), en la producción de forrajes de alta calidad sin el uso de fertilizantes sintéticos (Salazar-Sosa et al., 2007). Dichos abonos han demostrado efectos positivos en las características del suelo, en la disponibilidad de nutrientes para el desarrollo de los cultivos (Martínez-Blanco et al., 2013), y en la disminución de la contaminación derivada de los RO (Yadav et al., 2010) que contribuyen en la implementación de sistemas de producción limpia y ecológica (Ramos y Terry, 2014), pero poseen características profundamente variables (De Guardia et al., 2010).

La fracción líquida que se obtiene de los procesos de compostaje se conoce como extractos, tés y lixiviados de $\mathrm{CP}$ o de VC en el caso de humus de lombriz, y son considerados como fertilizantes líquidos orgánicos (FortisHernández et al., 2009), de los cuales existe muy poca información de su manejo en la nutrición de los cultivos (Vázquez et al., 2015). Salas y Ramírez (2001) puntualizaron la necesidad de desarrollar tecnologías adecuadas para la producción de abonos orgánicos de buena calidad que posibiliten su comercialización y correcta utilización en la agricultura, especialmente con lo referente a las concentraciones de nutrientes disponibles para las plantas. Con base en lo anterior, se estableció como objetivo de este trabajo comparar el efecto de la fertilización orgánica, inorgánica y su combinación, en la producción y contenido nutrimental del forraje de avena y en las propiedades fisicoquímicas del suelo.

\section{MATERIALES Y MÉTODOS}

El estudio se realizó en la Baja Babícora, principal zona productora de avena en México, en el Rancho Santa María localizado a 290 16' 13.2" N y $107^{\circ} 22^{\prime} 52.4^{\prime \prime}$ O, en Namiquipa, Chihuahua, con una altitud de $1998 \mathrm{~m}$, con temperatura media anual de $13{ }^{\circ} \mathrm{C}$ y precipitación promedio anual de 438.8 mm (Ramírez y Ávila, 1997).

El suelo utilizado fue de textura franco arcillo arenosa con $69 \%$ de arena y $26 \%$ de arcilla, con contenidos de $16,8,273,5503$ y $317 \mathrm{mg} \mathrm{kg}^{-1}$ de N-NO${ }_{3}=\mathrm{P}, \mathrm{K}^{+}, \mathrm{Ca}^{++} \mathrm{y} \mathrm{Mg}^{++}$, respectivamente, y 148, 177, 13 y $11 \mathrm{mg} \mathrm{kg}^{-1} \mathrm{de} \mathrm{Fe}^{++}, \mathrm{Mn}^{++}$, $\mathrm{Zn}^{++}$y $\mathrm{Cu}^{++}$, respectivamente; $4.7 \mathrm{~g} \mathrm{~kg}^{-1}$ de $\mathrm{MO}$, densidad aparente de $1.5 \mathrm{~g} \mathrm{~cm}^{-3}$, pH de 6.33 y CE de 4.90 mmhos $\mathrm{cm}^{-1}$, el cual no había sido cultivado durante los cinco años anteriores al estudio. Previo a la siembra, el suelo se preparó mediante un barbecho a $30 \mathrm{~cm}$ de profundidad con un arado de discos, y paso de rastra cruzado. La siembra de avena forrajera variedad Cevamex con una densidad de $300 \mathrm{~kg} \mathrm{ha}^{-1}$, se realizó el día 7 de febrero de 2015 con doble pasada de una sembradora John Deere de tipo chorro, que depositó la semilla a una profundidad de 4 a $5 \mathrm{~cm}$. Se aplicaron nueve riegos con intervalos de $10 \mathrm{~d}$.

Se utilizó un diseño completamente al azar, con cinco tratamientos de fertilización y seis repeticiones. La unidad experimental estuvo constituida por una parcela de un metro cuadrado. Los tratamientos consistieron en: $\mathrm{T} 1 \mathrm{C}$ : fertilización orgánica con compost (CP); T2-L: fertilización orgánica con lixiviados de vermicompost (VC); T3-Q: fertilización química; T4-QC: fertilización química más CP, y T5-QL: fertilización química más lixiviados de VC.

La dosis de CP empleada en la fertilización fue de 70 t ha-1 la cual fue dividida en tres aplicaciones que iniciaron al momento de la siembra y cada $30 \mathrm{~d}$. Esta dosis se calculó estimando la necesidad de MO del suelo utilizado para llevarlo a una condición media de acuerdo con lo recomendado por Castellanos et al. (2000), considerando el contenido inicial de $\mathrm{MO}$ y la textura del suelo, lo que da como resultado $23.0 \mathrm{~g} \mathrm{~kg}^{-1}$ de $\mathrm{MO}$ por incorporar. La dosis de fertilización con lixiviado de VC fue de $500 \mathrm{~L} \mathrm{ha}^{-1}$ en las dos primeras aplicaciones realizadas el 8 de febrero y el 1 de marzo, que aumentaron a $1000 \mathrm{~L} \mathrm{ha}^{-1}$ en las dos últimas aplicaciones realizadas el 30 de marzo y el 3 de mayo. La 
fertilización química consistió en $200 \mathrm{~kg} \mathrm{ha}^{-1}$ de 18N-46P$00 \mathrm{~K}$ el día 8 de febrero y $200 \mathrm{~kg} \mathrm{ha}^{-1}$ de $46 \mathrm{~N}-00 \mathrm{P}-00 \mathrm{~K}$ el 30 de marzo.

El CP y el VC empleados se obtuvieron a partir de una mezcla inicial de $\mathrm{RO}$ con una relación carbono/nitrógeno (C/N) de 25/1 (Hernández-Rodríguez et al., 2013). Para ello se utilizó estiércol de bovino de engorda como fuente de nitrógeno $(\mathrm{N})$, y como fuente de carbono aserrín de pino (Pinus) de partícula fina (menor de $2 \mathrm{~mm}$ ). La CP y VC se obtuvieron a las 24 semanas de transformación, en que se redujo su relación $\mathrm{C} / \mathrm{N}$ a niveles cercanos a 12/1, según las determinaciones de N-total y C-orgánico; además, presentaron valores superiores a $95 \%$ en la prueba del índice de germinación, según la prueba de Zucconi (1981), características que se han reportado como indicadores de madurez de los productos obtenidos mediante compostaje (Flavel y Murphy, 2006). El lixiviado se obtuvo del agua de riego usada en los canteros del VC. Las características de la CP y lixiviado de VC utilizados se muestran en el Cuadro 1.

Los muestreos de planta y suelo se realizaron a los 92 días después de la siembra (dds). En el muestreo de suelos se tomó de cada parcela, aproximadamente $500 \mathrm{~g}$ de suelo a una profundidad de 0 a $30 \mathrm{~cm}$. Dichas muestras se secaron a la sombra y posteriormente en estufa a $60{ }^{\circ} \mathrm{C}$ por $24 \mathrm{~h}$ y se tamizaron en malla No. 10 y 20. Las variables evaluadas en suelo fueron: MO por el método de WalkleyBlack; ${\mathrm{N}-\mathrm{NO}_{3}}^{=}$por el método de Brucina y espectrofotometría; P-aprovechable por Bray P1 (Bray y Kurtz, 1945); K+, $\mathrm{Ca}^{++}, \mathrm{Mg}^{++}$y Na${ }^{+}$, mediante acetato de amonio y su lectura

Cuadro 1. Características del compost y lixiviado de vermicompost y compost utilizados como fuente de fertilización orgánica.

\begin{tabular}{llcr}
\hline \multicolumn{2}{c}{ Característica } & $\begin{array}{c}\text { Lixiviado de } \\
\text { vermicompost }\end{array}$ & Compost \\
\hline $\mathrm{N}$-total & $\mathrm{g} \mathrm{kg}^{-1}$ & 16.0 & 17.2 \\
P-total & $\mathrm{g} \mathrm{kg}^{-1}$ & 0.42 & 2.1 \\
$\mathrm{~K}^{+}$ & $\mathrm{g} \mathrm{kg}^{-1}$ & 0.50 & 14.4 \\
$\mathrm{Ca}^{++}$ & $\mathrm{g} \mathrm{kg}^{-1}$ & 1.7 & 46.0 \\
$\mathrm{Mg}^{++}$ & $\mathrm{g} \mathrm{kg}^{-1}$ & - & 18.7 \\
$\mathrm{Na}^{+}$ & $\mathrm{g} \mathrm{kg}^{-1}$ & - & 2.9 \\
$\mathrm{Cu}^{++}$ & $\mathrm{mg} \mathrm{kg}^{-1}$ & 0.48 & 28.0 \\
$\mathrm{Fe}^{++}$ & $\mathrm{mg} \mathrm{kg}^{-1}$ & 2.47 & 2536.0 \\
$\mathrm{Mn}^{++}$ & $\mathrm{mg} \mathrm{kg}^{-1}$ & 0.65 & 367.0 \\
$\mathrm{Zn}^{++}$ & $\mathrm{mg} \mathrm{kg}^{-1}$ & 0.55 & 216.0 \\
$\mathrm{pH}$ & & 8.49 & 9.5 \\
$\mathrm{CE}$ & $\mathrm{mmhos} \mathrm{cm}^{-1}$ & 1.65 & 1.90 \\
\hline
\end{tabular}

en espectrofotómetro de absorción atómica (Perkin Elmer®, AAnalyst 100, New Jersey, USA); $\mathrm{Cu}^{++}, \mathrm{Fe}^{++}, \mathrm{Zn}^{++}$y $\mathrm{Mn}^{++}$mediante DTPA (Ácido dietilen triamino pentaacético) y su lectura en espectrofotómetro de absorción atómica (Perkin Elmer ${ }^{\circledR}$, AAnalyst 100, New Jersey, USA); pH con el uso de potenciómetro en dilución en agua 1:2 (p/v); densidad aparente (Da) con el método de la probeta, y conductividad eléctrica (CE) (Solu-brige, Beckman®, USA) en el extracto de saturación.

Las variables evaluadas en planta fueron: densidad de tallos por metro cuadrado mediante el conteo total de tallos en cada parcela; altura, medida en $\mathrm{cm}$ a partir de la base del tallo hasta el último nudo del mismo, de 20 plantas seleccionadas al azar de cada parcela, las cuales se cortaron aproximadamente a $1 \mathrm{~cm}$ de la superficie del suelo y pesaron para la evaluación de la producción de forraje verde. Se secaron a temperatura ambiente y posteriormente en estufa a $60{ }^{\circ} \mathrm{C}$ durante $24 \mathrm{~h}$ y se volvieron a pesar para estimar la producción de materia seca. Para el análisis nutricional se utilizaron las hojas completas, las cuales se molieron en un molino Wiley ${ }^{\circledR}$ (Thomas Scientific, USA) con malla No. 35. Se cuantificó: N-total por el método Kjeldahl (Digestor Novatech®, USA y Micro Kjeldahl Labconco ${ }^{\circledR}$, rapid destillation Unit, USA) (Fernández et al., 2006); $\mathrm{Ca}^{++}, \mathrm{Mg}^{++}, \mathrm{K}^{+}, \mathrm{Na}^{+}, \mathrm{Cu}^{++}, \mathrm{Fe}^{++}, \mathrm{Mn}^{++}$y $\mathrm{Zn}^{++}$mediante digestión con $25 \mathrm{~mL}$ de mezcla triácida $\left(\mathrm{HNO}_{3}, \mathrm{HClO}_{4}\right.$ y $\mathrm{H}_{2} \mathrm{SO}_{4}$ en relación 10:1:0.25) y espectrofotómetro de absorción atómica, y P-total mediante el método del vanadato-molibdato de amonio.

Los datos se analizaron con el procedimiento GLM del paquete estadístico SAS (Statistical Analisis System) versión 9.0. La comparación de medias se efectuó con la prueba de Tukey $(P \leq 0.05)$. Previo al análisis de variables se realizó la prueba de Levene para probar homogeneidad de varianzas. La varianza del error fue heterogénea únicamente para la variable $\mathrm{Na}$ en tejido foliar, la cual se analizó con estadísticas no paramétricas de Kruskal-Wallis.

\section{RESULTADOS Y DISCUSIÓN}

\section{Resultados en suelo}

Los análisis de varianza de las variables evaluadas en suelo se muestran en el Cuadro 2. En el contenido de MO sobresalieron estadísticamente los suelos fertilizados con fuentes orgánicas y la combinación T4-QC con valores en un rango de 17.0 a $18.1 \mathrm{~g} \mathrm{~kg}^{-1}$, lo que concuerda con lo reportado por Olivares-Campos et al. (2012) en que el efecto de la incorporación de compost y vermicompost puede remediar la carencia de MO de los suelos desde el primer año. 
La densidad aparente ( $\mathrm{Da}$ ) del suelo fue significativamente diferente entre tratamientos ( $P=0.013)$, obtuvo el valor más bajo, con $1.40 \mathrm{~g} \mathrm{~cm}^{-3}$, con el tratamiento $\mathrm{T} 1-\mathrm{C}$, lo que indica cierta relación entre los valores bajos de $\mathrm{Da}$ y altos de MO de los suelos estudiados (Meza y Geissert, 2003), lo que se ha relacionado con una disminución en la dureza e incremento en la porosidad del suelo (Hossne, 2008), lo que mejora su estructura y por ende la fertilidad natural del mismo (López-Mtz et al., 2001).

El contenido de $\mathrm{N}-\mathrm{NO}_{3}{ }^{~}$ en suelo no presentó diferencias estadísticas por efecto de los tratamientos, con valores entre 61 y $71 \mathrm{mg} \mathrm{kg}^{-1}$. Es importante mencionar que los tratamientos con fertilización orgánica igualaron al del fertilizante químico; además, se ha reportado que la utilización de abonos orgánicos contribuye al aporte de nutrientes, principalmente N (Fuentes et al., 2006), ya que la mayoría del $N$ que se encuentra en los suelos (98\%) se asocia con el material orgánico (Castellanos et al., 2000). Estos, resultados superaron lo encontrado por Fortis-Hernández (2009), quienes reportaron concentraciones de $\mathrm{N}-\mathrm{NO}_{3}=\mathrm{de}$ 50,49 y $35 \mathrm{mg} \mathrm{kg}^{-1}$ en suelos con fertilización química a base de N-P-K (200-100-100), $30 \mathrm{t} \mathrm{ha}^{-1}$, biocompost y $64 \mathrm{t}$ $\mathrm{ha}^{-1}$ de vermicompost, respectivamente.

Con relación al efecto de la fertilización en el contenido de P del suelo, los fertilizados con T1-C y T2-L presentaron los valores más altos con respecto al resto de los trata- mientos, a lo que Silva et al. (2006) indicaron que la acción de los microorganismos contenidos en los sustratos orgánicos influye directamente en la disponibilidad de nitrógeno y fósforo, de vital importancia para el mantenimiento y mejoramiento de la fertilidad y productividad del suelo.

El suelo con el tratamiento T1-C presentó los contenidos más altos de $\mathrm{K}^{+} \mathrm{y} \mathrm{Mg}^{++}$, lo que coincide con lo encontrado por Olivares-Campos et al. (2012) al incorporar $31 \mathrm{t} \mathrm{ha}^{-1}$ de compost al suelo, aun cuando se ha indicado que el $\mathrm{K}^{+}$ contenido en la $\mathrm{MO}$ es cuantitativamente poco importante dado que este elemento se encuentra principalmente asociado a la fracción arcillosa del mismo.

Se encontró diferencia significativa en el contenido de micronutrientes en los suelos según los diferentes tipos de fertilización, con excepción del $\mathrm{Fe}^{++}(\mathrm{P}=0.0510)$, como ya se mencionó anteriormente (Cuadro 2). Los valores más altos observados fueron: $242 \mathrm{mg} \mathrm{kg}^{-1}$ de $\mathrm{Mn}^{++}$con T2-L, 48 $\mathrm{mg} \mathrm{kg}^{-1}$ de $\mathrm{Zn}^{++}$con T1-C y $16 \mathrm{mg} \mathrm{kg}^{-1}$ de $\mathrm{Cu}^{++}$con T3-Q.

Los valores más altos para la variable $\mathrm{pH}$ se presentaron en los suelos fertilizados con T1-C, T2-L y T4-QC, lo que puede estar relacionado con el aporte de sales de $\mathrm{Ca}^{++} \mathrm{y}$ $\mathrm{Mg}^{++}$de las fuentes orgánicas empleadas. Fortis-Hernández et al. (2009) reportaron un incremento en los valores de pH respecto al valor inicial del suelo con la fertilización química a base de N-P-K (200-100-100) y fertilización orgánica a

Cuadro 2. Análisis de varianza del efecto de la fertilización orgánica, inorgánica y combinada sobre las propiedades fisicoquímicas del suelo.

\begin{tabular}{|c|c|c|c|c|c|c|c|c|c|}
\hline \multirow{2}{*}{ Variable } & \multicolumn{9}{|c|}{ Tratamientos } \\
\hline & $\mathrm{T} 1-\mathrm{C}$ & $\mathrm{T} 2-\mathrm{L}$ & T3-Q & $\mathrm{T} 4-\mathrm{QC}$ & T5-QL & $\mathrm{CV}$ & $\mathrm{R}^{2}$ & MS & $\mathrm{P}$ \\
\hline $\mathrm{MO}\left(\mathrm{g} \mathrm{kg}^{-1}\right)$ & $18.1 \mathrm{a}$ & $17.3 a$ & $13.3 b$ & $17.0 \mathrm{a}$ & $15.6 \mathrm{ab}$ & 13.2 & 0.4 & 4.60 & 0.0054 \\
\hline $\mathrm{Da}\left(\mathrm{g} \mathrm{cm}^{-3}\right)$ & $1.40 \mathrm{~b}$ & $1.50 \mathrm{a}$ & $1.51 \mathrm{a}$ & $1.47 \mathrm{ab}$ & $1.47 a b$ & 3.6 & 0.4 & 0.002 & 0.013 \\
\hline $\mathrm{pH}$ & $7.3 \mathrm{a}$ & $7.1 \mathrm{a}$ & $6.6 b$ & $7.1 \mathrm{a}$ & $6.7 b$ & 2.1 & 0.8 & 0.02 & $<0.0001$ \\
\hline $\mathrm{CE}\left(\mathrm{mmhos} \mathrm{cm}^{-1}\right)$ & $6.41 \mathrm{a}$ & $5.50 \mathrm{a}$ & $6.41 \mathrm{a}$ & $6.00 \mathrm{a}$ & $7.50 \mathrm{a}$ & 23.3 & 0.2 & 2.2 & 0.236 \\
\hline $\mathrm{N}-\mathrm{NO}^{3}=\left(\mathrm{mg} \mathrm{kg}^{-1}\right)$ & $70 a$ & $71 \mathrm{a}$ & $67 a$ & 61 a & $66 \mathrm{a}$ & 16.7 & 0.1 & 125 & 0.656 \\
\hline P-extraible $\left(\mathrm{mg} \mathrm{kg}^{-1}\right)$ & $17 a$ & $15 a$ & $7 \mathrm{~b}$ & $11 \mathrm{~b}$ & $10 \mathrm{~b}$ & 19.52 & 0.7 & 5.51 & $<0.0001$ \\
\hline $\mathrm{K}^{+}\left(\mathrm{mg} \mathrm{kg}^{-1}\right)$ & $412 \mathrm{a}$ & $279 a b$ & $308 a b$ & $333 a b$ & $233 b$ & 31.8 & 0.3 & 9290 & 0.053 \\
\hline $\mathrm{Ca}^{++}\left(\mathrm{mg} \mathrm{kg}^{-1}\right)$ & 5781 a & 5264 a & 5897 a & $5225 \mathrm{a}$ & $5152 \mathrm{a}$ & 9.6 & 0.3 & 276,175 & 0.058 \\
\hline $\mathrm{Mg}^{++}\left(\mathrm{mg} \mathrm{kg}^{-1}\right)$ & $362 a$ & $352 a b$ & $339 b$ & $339 b$ & $343 a b$ & 3.66 & 0.4 & 162 & 0.019 \\
\hline $\mathrm{Cu}^{++}\left(\mathrm{mg} \mathrm{kg}^{-1}\right)$ & $15 a b$ & $14 b$ & $16 a$ & $15 a b$ & $15 a b$ & 5.8 & 0.3 & 0.8 & 0.034 \\
\hline $\mathrm{Fe}^{++}\left(\mathrm{mg} \mathrm{kg}^{-1}\right)$ & 337 a & $313 a$ & $426 a$ & $320 \mathrm{a}$ & 381 a & 19.8 & 0.3 & 4956 & 0.051 \\
\hline $\mathrm{Mn}^{++}\left(\mathrm{mg} \mathrm{kg}^{-1}\right)$ & $222 a b$ & $242 \mathrm{a}$ & $200 a b c$ & $159 c$ & 168 bc & 16.1 & 0.5 & 1024 & 0.0005 \\
\hline $\mathrm{Zn}^{++}\left(\mathrm{mg} \mathrm{kg}^{-1}\right)$ & $48 \mathrm{a}$ & $34 a b$ & $29 \mathrm{~b}$ & $37 a b$ & $30 a b$ & 32.0 & 0.2 & 131 & 0.048 \\
\hline
\end{tabular}

Medias con letras iguales en las líneas no son estadísticamente diferentes (Tukey, P $\leq$ 0.05). MO: materia orgánica; Da: densidad aparente; CE: conductividad eléctrica; CV: coeficiente de variación (\%); MS: cuadrado medio; P. significancia del análisis de varianza; T1-C: fertilización orgánica con compost (CP); T2-L: fertilización orgánica con lixiviados de vermicompost (VC); T3-Q: fertilización química; T4-QC: fertilización química más CP;

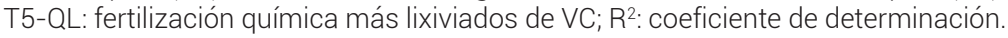


base de biocompost $\left(30 \mathrm{t} \mathrm{ha}^{-1}\right)$, después de 120 días de fertilización.

\section{Características del forraje de avena}

La mayor altura de planta se alcanzó con los tratamientos T3-Q, T4-QC y T5-QL, con valores entre 77.1 y $81.5 \mathrm{~cm}$ con diferencia significativa respecto al resto de los tratamientos, mientras que T2-L presentó el promedio más bajo con $44.6 \mathrm{~cm}$ (Cuadro 3). Estos resultados coinciden con los de Bar-Tal et al. (2004), quienes mencionaron que la incorporación de compost tiene un efecto positivo en los cultivos sólo cuando se realizan aplicaciones adicionales de $\mathrm{N}$ o cuando se realizan aplicaciones frecuentes durante varios años. Por otro lado, Fornaris y Rodríguez (2009) encontraron mayor estatura en plantas de tomate (Solanum lycopersicum L.) al ser fertilizadas con dosis de $500 \mathrm{~mL}$ de lixiviado de lombriz en $17 \mathrm{~L}$ de agua con respecto a dosis más bajas; al respecto, se ha mencionado que los niveles de ácidos húmicos y fúlvicos extraídos o producidos por los microorganismos presentes en el VC inducen el desarrollo vegetativo de las plantas (Arancon et al., 2007).

La producción de materia seca (MS) del forraje de avena fue significativamente mayor en las plantas fertilizadas con las combinaciones T4-QC y T5-QL y con fertilización química (T3-Q) con respecto a las fuentes orgánicas (Cuadro 3), lo cual pudo deberse a que es más eficiente el aprovechamiento de los dos tipos de fertilizantes aplicados a la vez, que cuando se aplican de manera individual. La misma tendencia fue encontrada por Flores-Aguilar et al. (2012), al fertilizar alfalfa (Medicago sativa) con fuente orgánica, inorgánica y su combinación con 28 t ha-1 de estiércol de ovino, $0.434 \mathrm{t}^{\text {ha-1 }}$ de superfosfato triple y $14 \mathrm{t}$ ha-1 de estiércol ovino junto con 0.217 t ha $^{-1}$ de superfosfato triple, respectivamente. En un trabajo realizado por Trujano et al. (2008), se encontró un rendimiento de 7078.90 $\mathrm{kg}$ de MS ha-1 en forraje de avena al aplicar estiércol más fertilización química. En investigaciones realizadas en la India y China se obtuvieron mejores cosechas de algodón
(Gossypium spp.), maíz (Zea mays L.), arroz (Oryza sativa), sorgo (Sorghum spp.) y trigo (Triticum spp.) cuando se usó una combinación de compost y fertilizantes minerales, que con la aplicación de dosis dobles de compost solo o de fertilizante mineral solo. En Bolivia, la producción de papa (Solanum tuberosum L.) se ha incrementado con el uso combinado de fertilizantes minerales y compost.

Aplicaciones combinadas han originado mayores producciones de materia seca por planta de tomate (Dalzell et al., 1991). Estos resultados son muy interesantes, tomando en cuenta que la aplicación de dosis excesivas de fertilizantes químicos al suelo pueden afectar también a la producción, ya que el rendimiento de un cultivo no aumenta proporcionalmente con la cantidad de nutrientes que se incorporan al suelo, sino a partir de una determinada concentración (López-Bermúdez, 2001).

Para la interpretación del contenido nutricional del forraje se utilizó el rango de suficiencia para el cultivo de avena en etapa de grano lechoso sugerido por Mills y Benton Jones, (1996). El contenido de N foliar se encontró dentro del rango de suficiencia por efecto de los tratamientos; sin embargo, el análisis estadístico arrojó dos grupos de significancia al presentar los valores más altos de las plantas fertilizadas con los tratamientos T3- $\mathrm{Q}$, T4-QC y T5-QL, con valores entre 20.8 y $24.6 \mathrm{~g} \mathrm{~kg}^{-1}$. Los resultados en el contenido de $\mathrm{N}$ observados en este estudio coinciden con lo reportado por Flores et al. (2014) quienes registraron concentraciones de 23.2 y $14.6 \mathrm{~g} \mathrm{~kg}^{-1}$ de $\mathrm{N}$ en follaje de avena al fertilizar con 63 $\mathrm{kg} \mathrm{ha}^{-1}$ de $\mathrm{N}$ con sulfato de amonio y con $1010 \mathrm{~m}^{3} \mathrm{ha}^{-1} \mathrm{de}$ biosólidos, respectivamente. Además, los valores de $\mathrm{N}$ total en el forraje obtenido con los tratamientos químico y combinados, equivalen a 13.0, 15.4 y $15.1 \%$ de proteína cruda (PC), superiores al $9.1 \%$ reportado por el NRC (2001) como adecuado para la etapa de corte en el forraje de avena. Valores similares fueron reportados por Trujano et al. (2008), quienes indicaron que la fertilización combinada con $9 \mathrm{t} \mathrm{ha}^{-1}$ más 180N-60P-00K, de abono orgánico y químico, respectivamente, produce una interacción adecuada que permite

Cuadro 3. Comparación de medias de características evaluadas en plantas de avena forrajera con fuentes de fertilización orgánica, inorgánica y su combinación.

\begin{tabular}{|c|c|c|c|c|c|c|c|c|c|}
\hline \multirow{2}{*}{ Variables } & \multirow[b]{2}{*}{$\mathrm{T} 1-\mathrm{C}$} & \multicolumn{6}{|c|}{ Tratamientos } & \multirow[b]{2}{*}{ MS } & \multirow[b]{2}{*}{$P$} \\
\hline & & $T 2-L$ & T3-Q & $\mathrm{T} 4-\mathrm{QC}$ & T5-QL & CV & $\mathrm{R}^{2}$ & & \\
\hline Altura de planta $(\mathrm{cm})$ & $60.60 \mathrm{~b}$ & $44.68 \mathrm{c}$ & $77.12 \mathrm{a}$ & $81.52 \mathrm{a}$ & $80.50 a$ & 9.2 & 0.9 & 40.3 & $<.0001$ \\
\hline Materia seca $\left(\mathrm{g} \mathrm{kg}^{-1}\right)$ & $233.1 \mathrm{~b}$ & $231.3 b$ & 396.6 a & 390.1 a & 476.8 a & 18.2 & 0.7 & 3960 & $<.0001$ \\
\hline Población (tallos m²) & 1072.50 & 757.50 & 1091.25 & 1038.75 & 1027.50 & & & & \\
\hline Producción de materia seca ( ha-1$\left.^{-1}\right)$ & $6.3 b$ & $2.5 b$ & $12.3 \mathrm{a}$ & $15.7 \mathrm{a}$ & $15.3 \mathrm{a}$ & 25.5 & 0.8 & 8.6 & $<.0001$ \\
\hline
\end{tabular}

Medias con letras iguales en las líneas no son estadísticamente diferentes (Tukey, P $\leq 0.05$ ). CV: coeficiente de variación (\%); MS: cuadrado medio; P. significancia del análisis de varianza; T1-C: fertilización orgánica con compost (CP); T2-L: fertilización orgánica con lixiviados de vermicompost (VC); T3-Q: fertilización química; T4-QC: fertilización química más CP; T5-QL: fertilización química más lixiviados de VC; R²: coeficiente de determinación. 
el aporte de los nutrimentos necesarios que demanda la planta para un buen forraje y alto contenido de PC.

Con respecto a la concentración de $\mathrm{P}$, todos los tratamientos se encontraron dentro del rango de suficiencia para avena reportado por Mills y Benton Jones (1996); sin embargo, las plantas que recibieron fertilización orgánica (T1-C y T2-L) presentaron los valores más altos, lo que supera lo encontrado por Trujano et al. (2008), quienes relacionaron contenidos de 1.6 y $1.4 \mathrm{~g} \mathrm{~kg}^{-1}$ de $P$ con la incorporación de $9 \mathrm{t} \mathrm{ha}^{-1}$ de estiércol y $3 \mathrm{t} \mathrm{ha}^{-1}$ de vermicompost, respectivamente, lo que significa una disminución de P cuando se aplicó mayor cantidad de abonos orgánicos. Además, Flores-Aguilar et al. (2012) mencionaron que cuando se eleva el pH del suelo de ácido a neutro o ligeramente alcalino, se logra una mayor tasa de mineralización del P, lo cual permite una mayor solubilidad y aprovechamiento de este nutriente, lo que coincide con lo observado en este trabajo.

Las concentraciones de $\mathrm{K}^{+}$encontrado en el tejido foliar de las muestras analizadas alcanzaron el nivel de suficiencia para avena, como efecto de los tratamientos, en los que se encontraron dos grupos de significancia en que los valores de los tratamientos orgánicos fueron los más bajos, los cuales se reportaron en trabajos previos de fertilización con estiércol vacuno y vermicompost con promedios de $23.5 \mathrm{~g} \mathrm{~kg}^{-1}$ de $\mathrm{K}^{+}$en el follaje de avena (Trujano et al., 2008).

Las medias registradas del contenido de $\mathrm{Na}^{+}$en tejido foliar fueron de 27.3, 23.8, 31.0, 30.3 y $31.3 \mathrm{~g} \mathrm{~kg}^{-1}$, para los tratamientos $T 1$ al $T 5$ respectivamente, los cuales no fueron diferentes estadísticamente $(P=0.142)$.

Con respecto a las concentraciones de micronutrientes en el forraje de la avena (Cuadro 4), se registraron contenidos superiores de $\mathrm{Mn}^{++}$y Fe ${ }^{++}$por efecto de los tratamientos T3-Q, T4-QC y T5-QL, respectivamente, pero inferiores para $\mathrm{Zn}^{++}$como respuesta de todos los tratamientos, según los rangos de suficiencia reportados como normales para este cultivo. Estos resultados coinciden parcialmente con los de Trujano et al. (2008), quienes reportaron que el uso combinado de fertilizantes químicos y abonos orgánicos provocaron concentraciones de nutrientes foliares en la avena por arriba de los estándares promedio. Se ha generalizado que la $\mathrm{MO}$ es una importante fuente de micronutrientes, los cuales se encuentran quelatados por las sustancias orgánicas, lo que favorece una adecuada nutrición de las plantas (Sierra y Rojas, 2015); sin embargo, Durán y Henríquez (2007) encontraron que a valores de pH cercanos o mayores a 7, como en el caso de los suelos de este estudio, es posible que el $\mathrm{Zn}^{++}$se mantenga en forma no soluble y acomplejado dentro del humus, por lo que la disponibilidad de los nutrientes en los abonos orgánicos no depende de su contenido total en el material, sino de la dinámica del proceso; así, algunos elementos pueden llegar a estar más disponibles por efecto del pH, de la humedad y de la aireación; o en los compostajes sin lombrices, por la temperatura alcanzada que permite el desarrollo de organismos termófilos especializados (Sierra y Rojas, 2015).

De acuerdo con el rango de suficiencia nutricional, con los tratamientos T3-Q y T5-QL se observó un estado nutricional más cercano a lo deseable para plantas de avena. Diferentes dosis y tipos de abonos orgánicos deben ser evaluados durante varios años de estudio.

\section{CONCLUSIONES}

Bajo las condiciones en que se realizó este estudio la incorporación de abonos orgánicos mejoró diversos indicadores de fertilidad del suelo durante el mismo ciclo de aplicación. Las fertilizaciones combinadas con fertilizantes químicos y orgánicos indujeron mayor altura de planta y producción de materia seca, lo que representa una alternativa viable para alcanzar niveles de producción óptima. La mejor respuesta en el contenido nutrimental foliar se obtuvo cuando se aplicó fertilizante químico y su combinación con lixiviado de vermicompost.

\section{AGRADECIMIENTOS}

Al Sr. Mario Montaño Córdova por facilitarnos los medios para la realización de esta investigación.

\section{BIBLIOGRAFÍA}

Arancon N. Q., C. A. Edwards, R. Dick and L. Dick (2007) Vermicompost tea production and plant growth impacts. Biocycle 48:51-52.

Bar-Tal A., U. Yermiyahu, J. Beraud, M. Keinan, R. Rosenberg, D. Zohar, V. Rosen and P. Fine (2004) Nitrogen, phosphorus and potassium uptake by wheat and their distribution in soil following successive, annual compost applications. Journal of Environmental Quality 33:1855-1865.

Bray R. H. and L. T. Kurtz (1945) Determination of total, organic and available forms of phosphorus in soils. Soil Science 59:39-45.

Butler D. M., N. N. Ranells, D. H. Franklin, M. H. Poore and J. T. Green (2007) Ground cover impacts on nitrogen export from manured riparian pasture. Journal of Environmental Quality 36:155-162.

Castellanos J. Z., J. X. Uvalle-Bueno y A. Aguilar-Santelises (2000) Manual de Interpretación de Análisis de Suelos y Aguas Agrícolas, Plantas y ECP. 2a ed. Colección INCAPA. Instituto de Capacitación para la Productividad Agrícola. Chapingo, México. 201 p.

Dalzell H. W., A. J. Biddlestone, K. R. Gray y K. Thurairajan (1991) Manejo del Suelo: Producción y Uso del Composte en Ambientes Tropicales y Subtropicales. Organización de las Naciones Unidas para la Agricultura y la Alimentación. Roma, Italia. 178 p.

De Guardia A., P. Mallard, C. Téglia, A. Marin, C. Le Pape, M. Launay, J. C. Benoist and C. Petiot (2010) Comparison of five organic wastes regarding their behaviour during composting: Part 1, biodegradability, stabilization kinetics and temperature rise. Waste Management 30:402-414.

Durán L. y C. Henríquez (2007) Caracterización química, física y microbiológica de vermicompostes producidos a partir de cinco sustratos orgánicos. Agronomía Costarricense 31:41-51. 


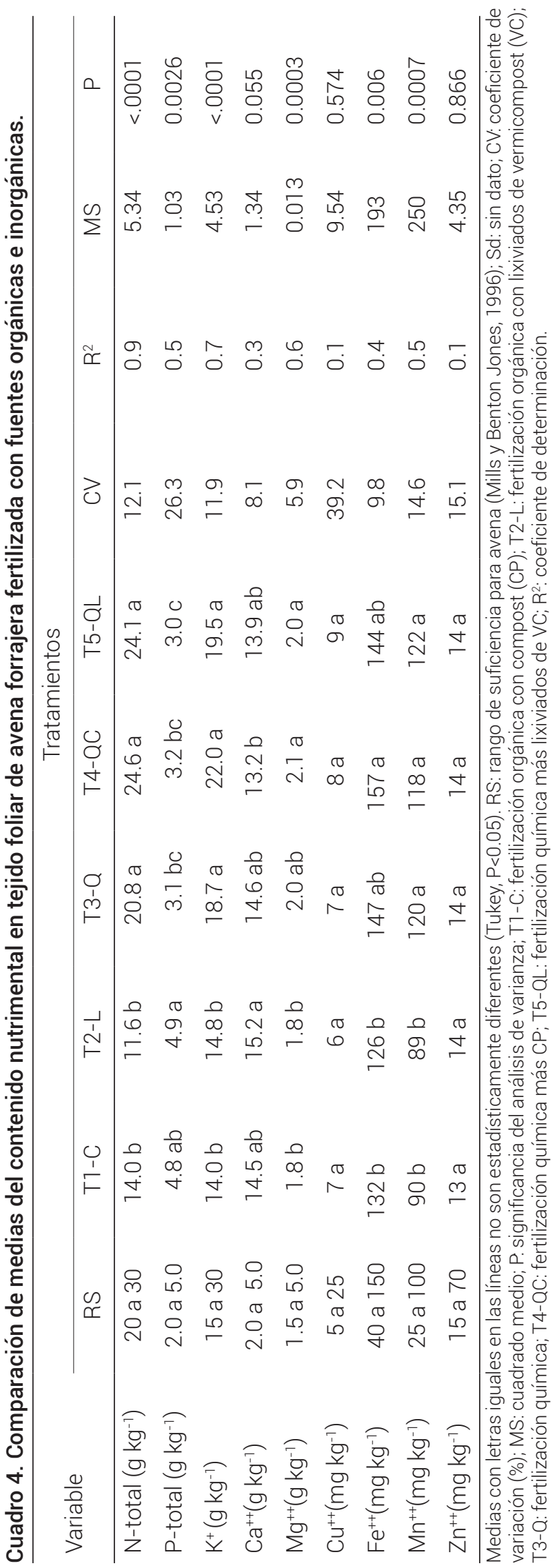

Fernández L. L. C., N. G. Rojas A., T. G. Roldán C. y M. E. Ramírez I. (2006) Manual de Técnicas de Análisis de Suelos Aplicadas a la Remediación de Sitios Contaminados. Secretaría de Medio Ambiente y Recursos Naturales, Instituto Nacional de Ecología. México, D.F. $180 p$

Flavel T. C. and D. V. Murphy (2006) Carbon and nitrogen mineralization rates after application of organic amendments to soil. Journal of Environmental Quality 35:183-193.

Flores-Aguilar J. J., R. Vázquez-Rosales, J. J. Solano-Vergara, V. Aguirre-Flores, F. I. Flores-Pérez, M. E. Bahena-Galindo, R. Oliver-Guadarrama, A. E. Granjeno-Colín y A. Orihuela-Trujillo (2012) Efecto de fertilizante orgánico, inorgánico y su combinación en la producción de alfalfa y propiedades químicas del suelo. Terra Latinoamericana 30:213-220.

Flores F. E., H. Moreno C., U. Figueroa V. y M. C. Potisek T. (2014) Disponibilidad de nitrógeno y desarrollo de avena forrajera (Avena sativa L.) con aplicación de biosólidos. Terra Latinoamericana 32:99105.

Flotats R. X. y F. Solé M. (2008) Situación actual en el tratamiento de los residuos orgánicos: aspectos científicos, económicos y legislativos. In: Compostaje. J. Moreno C. y R. Moral H. (eds.). MundiPrensa. Madrid, España. pp:43-74.

Fornaris S. A. y P. A. Rodríguez F. (2009) Influencia de dosis crecientes de lixiviados de abonos mixtos microbianos y lixiviado humus de lombriz sobre algunas variables morfoagronómicas en el cultivo de tomate (Lycopersicum esculentum Mill). Ciencia en su PC 2:100-114

Fornes F., D. Mendoza-Hernández, R. García-de-la-Fuente, M. Abad and R. M. Belda (2012) Composting versus vermicomposting: a comparative study of organic matter evolution through straight and combined processes. Bioresource Technology 118:296-305.

Fortis-Hernández M., J. A. Leos-Rodríguez, P. Preciado-Rangel, I. OronaCastillo, J. A. García-Salazar, J. L. García-Hernández y J. A. OrozcoVidal (2009) Aplicación de abonos orgánicos en la producción de maíz forrajero con riego por goteo. Terra Latinoamericana 27:329-336

Fuentes B., N. Bolan, R. Naidu and M. L. Mora (2006) Phosphorus in organic waste-soil systems. Journal of Soil Science and Plant Nutrition 6:64-83.

Hernández-Rodríguez 0. A., A. Hernández-Tecorral, C. Rivera-Figueroa, A. M. Arras-Vota y D. Ojeda-Barrios (2013) Calidad nutrimental de cuatro abonos orgánicos producidos a partir de residuos vegetales y pecuarios. Terra Latinoamericana 31:35-46.

Hossne G. A. J. (2008) La densidad aparente y sus implicaciones agrícolas en el proceso expansión/contracción del suelo. Terra Latinoamericana 26:195-202.

López-Bermúdez F. (2001) El riesgo de desertificación. In: Agricultura y Desertificación. F. M. de Santa Olalla M. (ed.). Mundi-Prensa. Madrid, España. pp:15-38.

López-Mtz. J. D., A. Díaz E., E. Martínez R. y R. D. Valdez C. (2001) Abonos orgánicos y su efecto en propiedades físicas y químicas del suelo y rendimiento en maíz. Terra Latinoamericana 19:293-299.

Lübke U. y S. Lübke (2008) Materia orgánica como un recurso para la fertilidad del suelo. In: II Conferencia Internacional sobre EcoBiología del Suelo y el Compost. 26-29 de noviembre 2008 Puerto de la Cruz, Tenerife, España. pp:309-316.

Márquez-Hernández C., P. Cano-Ríos, U. Figueroa-Viramontes, J. A. Avila-Diaz, N. Rodríguez-Dimas y J. L. García-Hernández (2013) Rendimiento y calidad de tomate con fuentes orgánicas de fertilización en invernadero. Phyton (Buenos Aires) 82:55-61

Martínez-Blanco J., C. Lazcano, T. Christensen, P. Muñoz, J. Rieradevall, J. Møller, A. Antón and A. Boldrin (2013) Compost benefits for agriculture evaluated by life cycle assessment. A review. Agronomy for Sustainable Development 33:721-732.

Meza P. E. y D. Geissert K. (2003) Estructura, agregación y porosidad en suelos forestales y cultivados de origen volcánico del Cofre de Perote, Veracruz, México. Foresta Veracruzana 5:57-60.

Mills H. A. and J. Benton Jones (1996) Plant Analysis Handbook II: A Practical Sampling, Preparation, Analysis, and Interpretation Guide. Micro-Macro Publishing, Inc. Athens, Georgia. 422 p.

NRC, National Research Council (2001) Nutrient Requirements of Dairy Cattle. 7th ed. National Academy Press. Washington, D. C. 408 p.

Olivares-Campos M. A., A. Hernández-Rodríguez, C. Vences-Contreras, J. L. 
Jáquez-Balderrama y D. Ojeda-Barrios (2012) Lombricomposta y composta de estiércol de ganado vacuno lechero como fertilizantes y mejoradores de suelo. Universidad y Ciencia. Trópico Húmedo 28:27-37.

Ramírez L. M. R. y M. R. Ávila M. (1997) El agroecosistema temporalero de la baja Babícora y regiones similares: su estructura actual, algunos de sus biocomponentes y su diseño futuro. Folleto Científico No. 3. Campo Experimental Sierra de ChihuahuaCentro de Investigación Regional del Norte Centro-INIFAP, Cd. Cuauhtémoc, Chihuahua. $46 \mathrm{p}$.

Ramírez-Ordóñes S., D. Domínguez-Díaz, J. J. Salmerón-Zamora, G. Villalobos-Villalobos y J. A. Ortega-Gutiérrez (2013) Producción y calidad del forraje de variedades de avena en función del sistema de siembra y de la etapa de madurez al corte. Revista Fitotecnia Mexicana 36:395-403.

Ramos A. D. y E. Terry A. (2014) Generalidades de los abonos orgánicos: importancia del bocashi como alternativa nutricional para suelos y plantas. Cultivos Tropicales 35:52-59.

Salas E. y C. Ramírez (2001) Bioensayo microbiano para estimar los nutrimentos disponibles en los abonos orgánicos: calibración en el campo. Agronomía Costarricense 25:11-23.

Salazar-Sosa E., H. I. Trejo-Escareño, C. Vázquez-Vázquez y J. D. López-Martínez (2007) Producción de maíz bajo riego por cintilla, con aplicación de estiércol bovino. Phyton (Buenos Aires) 76:169-185.
SIAP, Sistema de Información Agroalimentaria y Pesquera (2015) Anuario Estadístico de la Producción Agrícola. Sistema de Información Agroalimentaria y Pesquera, SAGARPA, México. http://infosiap. siap.gob.mx/aagricola_siap_gb/icultivo/index.jsp. (Agosto 1, 2107)

Sierra B. C. y C. Rojas W. (2015) La Materia Orgánica y su Efecto en las Características Físico-químicas y Biológicas del Suelo. INIA. Ministerio de Agricultura. Santiago, Chile. 22 p.

Silva P. A., D. M. Coral E. y J. C. Menjivar S. (2006) Efecto de la fertilización nitrogenada sobre la actividad microbial y rendimiento de avena forrajera en un suelo andisol del departamento de Nariño, Colombia. Acta Agronómica 55:2-12

Trujano S. L. D., A. González P., J. Jaimes J., J. A. Cueto W. y J. R. Hernández S. (2008) Evaluación de fertilizantes sobre la avena forrajera. Revista Chapingo Serie Zonas Áridas 7:73-82.

Vázquez V. P., M. Z. García L., M. C. Navarro C. y D. García H. (2015) Efecto de la composta y té de composta en el crecimiento y producción de tomate (Lycopersicon esculentum Mill.) en invernadero. Revista Mexicana de Agronegocios 36:1351-1356.

Yadav K. D., V. Tare and M. M. Ahammed (2010) Vermicomposting of source-separated human faeces for nutrient recycling. Waste Management 30:50-56

Zucconi F., A. Pera, M. Forte and M. De Bertoldi (1981) Evaluating toxicity of immature compost. Biocycle 22:54-57. 